\title{
Isolation and Identification of the Pathogen of Cardamine violifolia Bacterial Blight Disease in China
}

\author{
Jinping Wu, Zhenbiao Jiao, Leifu Chen, Zhengming Qiu and Fengling Guo* \\ Hubei Academy of Agricultural Sciences, Wuhan 430064, China \\ *Corresponding author
}

\section{A B S T R A C T}

Keywords

Cardamine violifolia,

Bacterial blight,

Pseudomonas sp.,

$16 \mathrm{~S}$ rDNA sequences.

Article Info

Accepted:

12 November 2016

Available Online:

10 December 2016
A bacteria disease was observed on Cardamine violifolia in production base, enshicity, Hubei Province, China in 2015. The disease mainly happened on Cardamine violifolia leaves. Four strains were isolated from the diseased Cardamine violifolia leaves. And the same strain was re -isolated from the inoculated leaves by the Koch's rule. The similar symptom was produced onhealthy leaves after inoculating the strain. The pathogen was identified as Pseudomonas sp. according to the characters of cultural colonies, results of 16S rDNA sequences analysis (NCBI acc. no.1918730)

\section{Introduction}

Cardamine violifolia is found in Yutangba, Shuanghe Town (an area rich in Se) of Enshi city, Hubei province, China, has strong capacity of accumulating Se .The Se content in seedling leaves exceeds $1000 \mathrm{mg} / \mathrm{kg}$, which is the international standard of Sehyperaccumulator (Karol et al., 2015). So Cardamine violifolia was artificial cultivation. A bacterial disease was found on leaves of Cardamine violifolia grown under greenhouse in winter of 2014 and 2015. The disease usually started from leaf edges near hydathodes and quickly enlarged to form Vshaped lesions frequently surrounded by chlorotic areas. These lesions finally coalesced to form the severe blight along the whole leaves.
The objective of this research was to identify the causal agent of Cardamine violifolia disease suspected to be bacterial blight disease.

\section{Materials and Methods}

\section{Isolation of bacteria causing bacterial blight disease}

Blight disease was observed on leaves of Cardamine violifolia (Fig.1). The pathogens were isolated by tissue segment method (Rangaswami. 1958) on nutrient agar (NA: 3 $\mathrm{gl}^{-1}$ beef extract, $5 \mathrm{gl}^{-1}$ peptone, $17 \mathrm{gl}^{-1}$ agar; $\mathrm{pH}$ 7.2) medium. Infected Cardamine violifolia leaves were cut into small pieces 
of $1.0 \sim 1.5 \mathrm{~cm}$, surface sterilized with $0.1 \%$ mercuric chloride for $1 \mathrm{~min}$, and washed in sterile distilled water thrice and blotted dry with sterilized filter paper. Then the leaf bits were placed in Petri plates containing NA. The plates were incubated at $28 \pm 2{ }^{\circ} \mathrm{C}$ for 24 48 h. Colony grew around the tissue mass, which were aseptically moved by using an inoculation loop and transferred to $\mathrm{NA}$ at $28 \sim 30{ }^{\circ} \mathrm{C}$ for $24 \sim 48 \mathrm{~h}$, discrete bacterial colonies were removed by using an inoculation loop, re-streaked on NA and incubated aerobically for 24 48 h. Individual colonies were isolated, subcultured twice to ensure purity and then stored in $15 \%$ sterilized glycerol at $-80^{\circ} \mathrm{C}$.

\section{Pathogenicity tests}

The bacterial suspension of $1 \times 10^{8} \mathrm{CFU} \cdot \mathrm{ml}^{-1}$ prepared in sterile distilled water, woundinoculated and then incubated at $28 \pm 2{ }^{\circ} \mathrm{C}$ and kept in humid conditions (Fang. 1996). Plants used as negative controls were inoculated with the sterile distilled water. Blight disease symptoms were observed every 12 hours. Each treatment repeated for three times.

\section{Phylogenic characterization}

The sequence of the $16 \mathrm{~S}$ rDNA was obtained from the total of the pathogen by PCR amplification with sense primer 5'AGAGTTTGATCCTGGCTCAG-3' and antisense primer 5'-CGGCTACCTTGT TACGACTTC-3' (Weisburg et al., 1991). The PCR amplification conditions were as follows: one denaturation step (3min at 95 $\left.{ }^{\circ} \mathrm{C}\right), 35$ cycles of amplification $\left(30 \mathrm{~s}\right.$ at $95{ }^{\circ} \mathrm{C}$, 30 s at $50{ }^{\circ} \mathrm{C}, 1.5 \mathrm{~min}$ at $72{ }^{\circ} \mathrm{C}$ ), and a final elogation step of $10 \mathrm{~min}$ at $72{ }^{\circ} \mathrm{C}$. The PCR products were checked by electrophoresis on $1.0 \%$ agarose gels, and then the corresponding products were purified with an AxyPrepTM DNA gel extraction kit
(Axygen Scientific, Inc, USA). Purified products were ligated into pGEM-T vectors (Promega Co., China), and then transformed into E. coli DH-5acells. Positive colonies were selected by the blue-white screening procedure (Sambrook et al., 1989). The bacteria for seeking out was cultured $12 \mathrm{~h}$ in liquid $\mathrm{LB}$ culture, $37^{\circ} \mathrm{C}, 200 \mathrm{r} \bullet \mathrm{min}^{-1}$, and then amplified by using a pair of primers pUC/M13, detected by gel electrophoresis and confirmed to contain the fragment. Positive clones were sequenced (BGI Co., Beijing, China) and the obtained sequences were compared with the sequences in Genbank. The $16 \mathrm{~S}$ rDNA sequences of other related bacteria were obtained from GenBank and BLASTN with the pathogen. Phylogenic analysis was using the Molecular Evolutionary Genetics Analysis (MEGA) Software Version 5.1.

\section{Results and Discussion}

\section{Pathogenicity tests}

Individual colonies were achieved by using an inoculation loop. The bacteria strain was round, smooth, protruding. It was Gramnegative, rod shaped, not endosporeforming. Water-soaked lesions first appeared on the wounds after 3d, the Blight disease on leaves in 7d (Fig.2). Re-isolation from inoculated leaves yielded colonies that consistent with the pathogen tester isolates. The repeated experiments confirmed the same results.

\section{Phylogenic characterization}

The partial $16 \mathrm{~S}$ rDNA sequence containing $1,419 \mathrm{bp}$ nucleotides (Accession nos: 1918730) was aligned with all related sequences in the NCBI database by the BLASTN program. The sequence identity among the sample and other Pseudomonas sp.species was $100 \%$. The phylogenetic tree 
of the strain and the other Pseudomonas species in Fig.3 shows that the pathogenic
16S rDNA region and Pseudomonas sp. comprise the same cluster.

Fig.1 Natural symptoms of Cardamine violifolia bacterial blight disease.

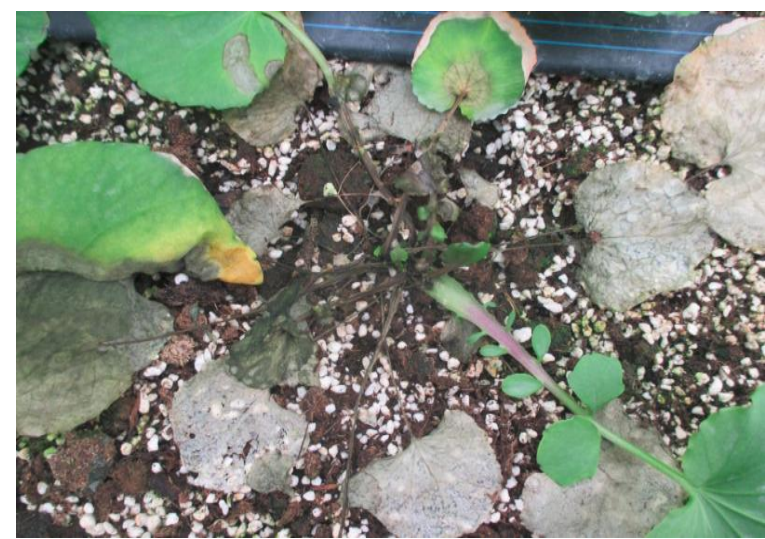

Fig.2 The symptoms of Cardamine violifolia at 7d post-inoculation.

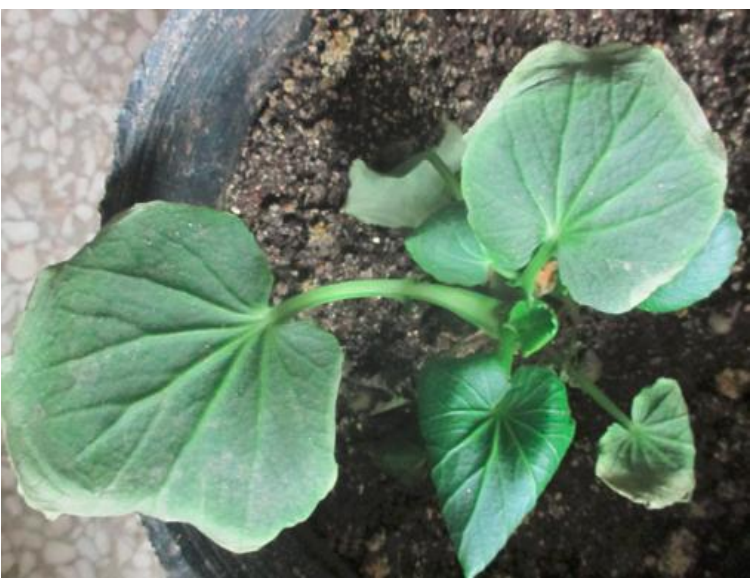

Fig.3 Phylogenetic tree showing the position of Pseudomonas sp. based on 16S rDNA sequences using neighbour-joining method. Bacillus subtilis 2BS2 (HM185057) was used as an outgroup

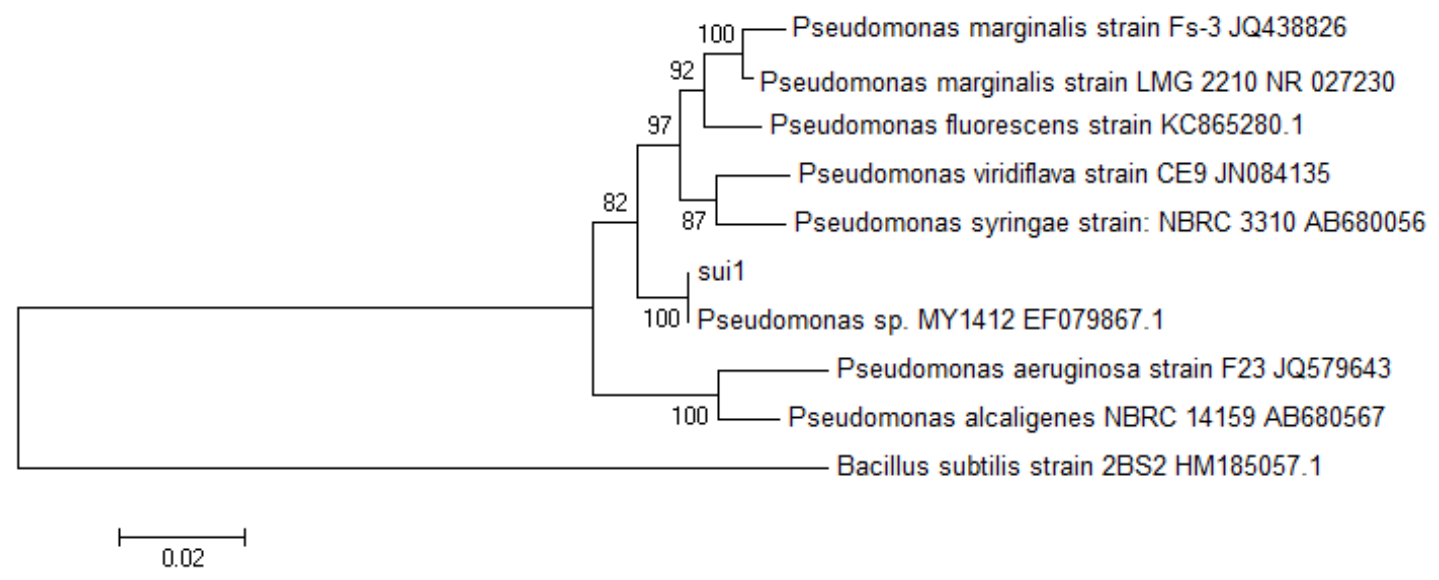


Classification standard of $16 \mathrm{~S}$ rDNA sequence indicates that the same species share higher than $97 \%$ of sequence identity each other. So, it can preliminary predicate that the strain is Pseudomonas sp.

To our knowledge, this is the first report of Pseudomonas sp. the causal agent of Cardamine violifolia bacterial blight in Enshi city, Hubei province, China. To prevent the spread of the disease to other regions, firstly, characterization of potential host range of the pathogen and after then the evaluation of Cardamine violifolia cultivars and lines for their reaction to the pathogen strains for putative sources of resistance are recommended.

\section{Acknowledgement}

This work was financially supported by The Modern Agricultural Industry Technology System Project Supported by Ministry of Agriculture [Grant No. nycytx-35-02-06].

\section{References}

Fang, Z.D. 1996. The research methods of plant disease. China Agriculture Press, 179.

Karol, M., Matúš, K., Ihsan, A.A. 2015. Lectotypification of names of Himalayan Brassicaceae taxa currently placed in the genus Cardamine. PhytoKeys, 50: 9-23

Rangaswami, G. 1958. An agar block technique for isolating soil micro organisms with special reference to Pythiaceous fungi. Sci. Cult., 24: 85.

Sambrook, J., Fritsch, E.F., Maniatis, T. 1989. Molecular cloning: a laboratory manual (Second Edition). Cold Spring Harbor Laboratory Press, Cold Spring Harbor, N.Y.

Weisburg, W.G., Barns, S.M., Pelletier, D.A., Lane, D.J. 1991. 16S ribosomal DNA amplification for phylogenetic study. J. Bacteriol., 173: 697-703.

\section{How to cite this article:}

Jinping Wu, Zhenbiao Jiao, Leifu Chen, Zhengming Qiu and Fengling Guo. 2016. Isolation and Identification of the Pathogen of Cardamine violifolia Bacterial Blight Disease in China. Int.J.Curr.Microbiol.App.Sci. 5(12): 203-206. doi: http://dx.doi.org/10.20546/ijcmas.2016.512.022 